\title{
COVID-19: Perfil clínico de pacientes em um hospital do Estado de Sergipe
}

COVID-19: Clinical profile of patients in a hospital in the State of Sergipe

COVID-19: Perfil clínico de los pacientes de un hospital del Estado de Sergipe

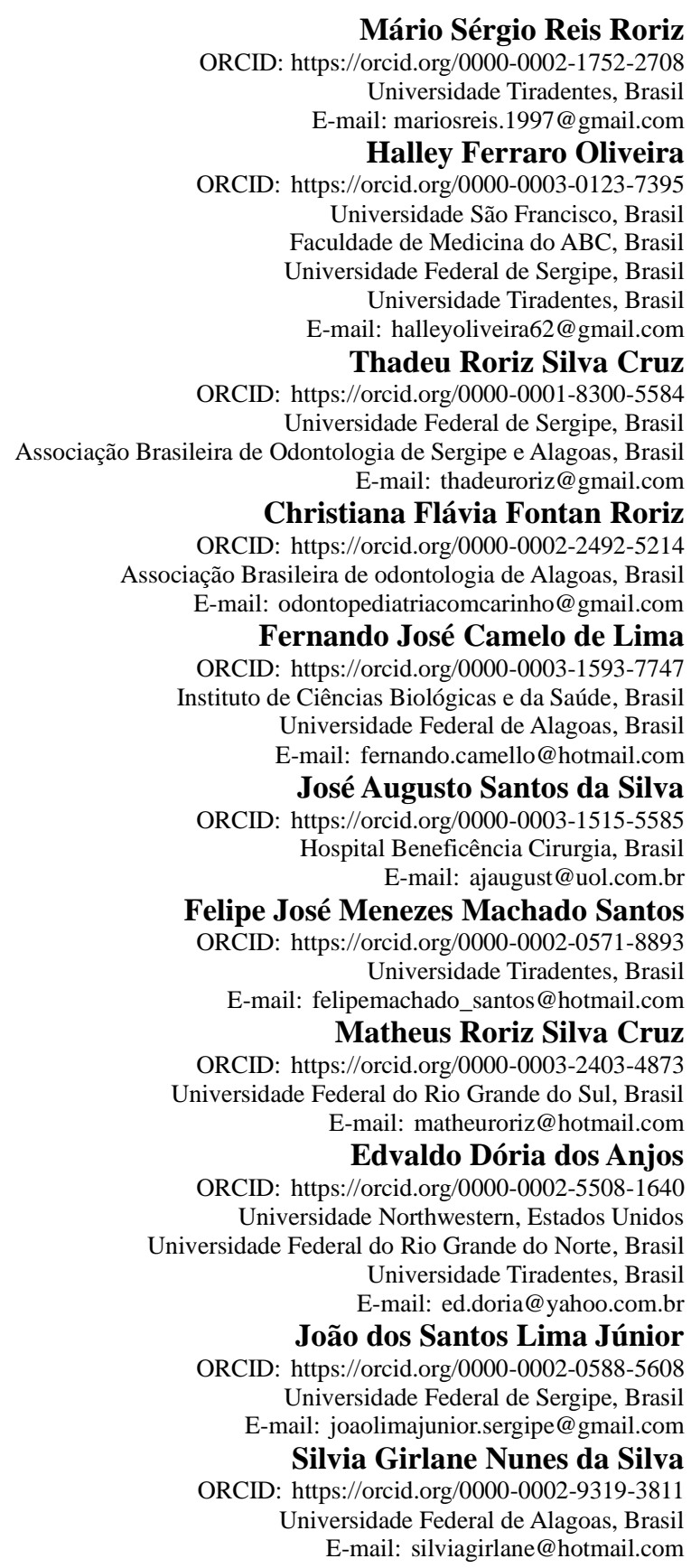

\section{Resumo}

Devido ao recente surto do coronavírus, milhões de pessoas foram atingidas mundialmente. Os sintomas como febre, tosse, fadiga e dispneia são mais prevalentes em pacientes com comorbidades. O objetivo deste estudo foi realizar um levantamento epidemiológico do quadro clínico e comorbidades em pacientes com COVID-19 em estado grave e não grave em um hospital do Estado de Sergipe. Foi realizado um estudo descritivo, coletando dados de prontuários de pacientes infectados por COVID-19 do Hospital público Regional de Itabaiana Dr. Pedro Garcia Moreno, localizado 
em Itabaiana por um período de 3 meses. O total da amostra foi de 64 voluntários, sendo divididos em dois grupos de 32 pacientes, sendo um grupo de pacientes em estado não grave (GC) e outro grupo de pacientes em estado grave (GT). A idade média do presente estudo foi de 58.9 anos, sendo o sexo masculino $(68.5 \%)$ e a etnia feoderma $(62.5 \%)$, os mais prevalentes. A pressão arterial média sistólica (PAS) foi de $122 \mathrm{mmHg}$ e a pressão arterial média diastólica (PAD) foi de $79.8 \mathrm{mmHg}$. Os sintomas presentes foram astenia $(90.63 \%)$, dispneia $(84.38 \%)$, cefaleia $(73.44 \%)$, tosse $(68.8 \%)$, febre $(65.6 \%)$, disgeusia $(54.7 \%)$, dor de garganta $(6.26 \%)$ e diarreia $(4.7 \%)$. As comorbidades mais prevalentes foram hipertensão arterial sistêmica (HAS) de $42.8 \%$ e Diabetes Mellitus (DM) de 15.63\%. Conclui-se que a etnia mais acometida foi a feoderma, o sintoma mais comum em ambos os grupos (GT e GC) foi a astenia, e a diarreia, o sintoma menos prevalente em ambos os grupos. As comorbidades mais prevalentes foram HAS e DM.

Palavras-chave: Epidemiologia; Coronavírus; Comorbidades.

\begin{abstract}
Due to the recent outbreak of the coronavirus, millions of people have been affected worldwide. Symptoms such as fever, cough, fatigue and dyspnea are more prevalent in patients with comorbidities. Conduct a reserch of epidemiological, clinic symptons and comorbidities in patients with COVID-19 in serious and mild conditions in a Hospital in the state of Sergipe. A descriptive study was performed, through a data collection from records of patients infected with COVID-19 at the Regional public Hospital of Itabaiana Dr. Pedro Garcia Moreno, located in Itabaiana, in a period of three months. The total sample was 64 volunteers, being divided into two groups of 32 patients, one group in a mild condition (GC) and another group in a serious condition (GT) The mean age of the present study was 58.3 years, with males $(68.5 \%)$ and pheoderm ethnicity $(62.5 \%)$ being more prevalent. The average systolic blood pressure (SBP) was $122 \mathrm{mmHg}$ and the average diastolic blood pressure was $79.8 \mathrm{mmHg}$. The present symptoms was asthenia $(90.63 \%)$, dyspnea $(84.38 \%)$, headache $(73.44 \%)$, cough $(68.8 \%)$, fever $(65.6 \%)$, dysgeusia $(54.7 \%)$, sore throat $(6.26 \%)$ and diarrhea $(4.7 \%)$. The most prevalent comorbidities were Systematic Arterial Hypertension (SAH) of $42.8 \%$ and Diabetes Mellitus (DM) of $15.63 \%$. It is concluded that the most affected ethnicity was pheoderm, the most common symptom in both groups (GT and GC) was asthenia and diarrhea was the least prevalent symptom in both groups. The most prevalent comorbidities were SAH and DM.
\end{abstract}

Keywords: Epidemiology; Coronaviruses; Comorbidities.

\title{
Resumen
}

Debido al reciente brote del coronavirus, millones de personas se han visto afectadas en todo el mundo. Los síntomas como fiebre, tos, fatiga y disnea son más prevalentes en pacientes con comorbilidades. El objetivo de este estudio fue realizar una encuesta epidemiológica del cuadro clínico y las comorbilidades en pacientes con COVID-19 en condiciones graves y no graves en un hospital del Estado de Sergipe. Se realizó un estudio descriptivo, recolectando datos de historias clínicas de pacientes infectados por COVID-19 del Hospital Público Regional de Itabaiana Dr. Pedro García Moreno, ubicado en Itabaiana por un período de 3 meses. La muestra total estuvo conformada por 64 voluntarios, divididos en dos grupos de 32 pacientes, un grupo de pacientes en condición no severa (GC) y otro grupo de pacientes en condición severa (GT). La edad media del presente estudio fue de 58,9 años, siendo los hombres $(68,5 \%)$ y la etnia feodermo $(62,5 \%)$ la más prevalente. La presión arterial sistólica (PAS) media fue de $122 \mathrm{mmHg}$ y la presión arterial diastólica (PAD) media fue de 79,8 mmHg. Los síntomas presentes fueron astenia $(90,63 \%)$, disnea $(84,38 \%)$, cefalea $(73,44 \%)$, tos $(68,8 \%)$, fiebre $(65,6 \%)$, disgeusia $(54,7 \%)$, dolor de garganta $(6,26 \%)$ y diarrea $(4,7 \%) . \%)$. Las comorbilidades más prevalentes fueron la hipertensión arterial sistémica (HSA) del 42,8\% y la diabetes mellitus (DM) del 15,63\%. Se concluye que la etnia más afectada fue la feodermia, el síntoma más común en ambos grupos (TG y GC) fue la astenia y la diarrea, el síntoma menos prevalente en ambos grupos. Las comorbilidades más prevalentes fueron HSA y DM.

Palabras clave: Epidemiología; Coronavirus; Comorbilidades.

\section{Introdução}

A COVID-19 é uma doença causada pelo coronavírus, um vírus da família coronaviridae que contém uma larga fita simples de RNA envelopado, podendo não somente infectar humanos mais também uma vasta variedade de animais (Velavan \& Meyer, 2020).

O recente surto do novo coronavírus teve um grande impacto mundial, atingindo milhões de pessoas. Os danos não se limitaram à saúde pública e, sim, ao aspecto econômico, científico e político devido aos financiamentos e descoberta de vacinas (Khan et al., 2021). O novo coronavírus é a grande preocupação internacional, apesar de sua origem zoonótica, pode se tornar fatal em humanos. A maioria dos pacientes em hospitais na China apresentaram infecção severa no trato respiratório inferior na forma de pneumonia (Deshmukh et al., 2020).

A etiopatogenia da COVID-19 pode ser explicada através da ligação da proteína S1 e S2 (spin protein) do vírus 
SARS-Cov-2 com receptores das células do hospedeiro através da enzima conversora de angiotensina 2 (ACE-2) (Raghuvamsi et al., 2020). Sua taxa de transmissão é de 18.7 vezes maior em ambientes fechados (Bulfone et al., 2021).

Os principais sintomas da COVID-19 consistiam em febre, tosse, fadiga e dispneia, sendo mais prevalentes em pacientes como comorbidades como obesidade, hipertensão, diabetes, doença cardiovascular, doença pulmonar obstrutiva crônica, câncer e doença renal crônica (Badawi \&Vasileva, 2021). Adil et al. (2020) afirmaram que 80\% das infecções são leves a moderadas, incluindo casos com e sem pneumonia, já os casos severos correspondem a $13 \%$ dos infectados, sendo que $6,1 \%$ dos pacientes necessitam de cuidados intensivos.

A estratégia terapêutica atual da COVID-19, em sintomáticos, consiste em prevenção infecciosa com oseltamivir/ antibióticos intravenosos, suporte clínico em pacientes graves com oxigênio e suporte ventilatório mecânico (Li et al., 2020). Além dessa estratégica terapêutica, variando de acordo com as complicações, corticoide, interferon beta, anticoagulantes, tocilizumabe, podem ser utilizados em combate à COVID-19 (Barry et al., 2021). O objetivo desse trabalho é realizar o levantamento epidemiológico, avaliação de quadro clínico e comorbidades envolvidas em pacientes com COVID-19 em estados graves e não graves em um hospital do estado de Sergipe.

\section{Material e Métodos}

Foi realizado um estudo descritivo (Rodrigues et al., 2021) nos setores de unidades de internamento com enfermarias e Unidades Intensivas do Hospital público Regional de Itabaiana Dr. Pedro Garcia Moreno, localizado em Itabaiana, Sergipe, conforme projeto aprovado junto ao Comitê de Ética e Pesquisa número CAAE 31540520.2.00005546.

Para a execução do levantamento epidemiológico, foram utilizados todos os EPIs em comuns recomendados pela Organização Mundial de Saúde, Ministério da Saúde e SCIH do hospital como aventais descartáveis de manga longa, punho com elástico, gramatura superior a 30, gorros, óculos ou protetores faciais Face Shield, máscaras PFF2 ou N95, luvas estéreis descartáveis, ficha clínica própria desenvolvida para pesquisa (cópia em anexo), lanterna auxiliar e acesso ao prontuário da paciente para coleta de dados da pesquisa.

Todo material necessário para realização da pesquisa foi adquirido por conta dos pesquisadores, inclusive os EPIs serão de responsabilidade deles, não poderão, portanto, utilizar os EPIs do hospital, considerando a situação atual de pandemia, garantindo seu uso aos profissionais que prestam a assistência direta ao paciente.

Durante três meses para a coleta de dados, de acordo com o cronograma de atividades, foram examinados pacientes com COVID-19 internados com Síndromes Respiratórias Agudas (SRAG) em estado graves e não graves no hospital, sendo que alguns critérios foram seguidos:

a. Foram considerados pacientes em estado graves - aqueles que já foram ou estejam entubados;

b. Foram considerados pacientes em estado não graves - aqueles que não receberam indicações de entubação;

c. Nesta pesquisa a amostra foi de 64 voluntários, divididos em dois grupos de 32 pacientes: um grupo teste, que recebeu indicações de intubação e um grupo controle, composto por pacientes que não receberam indicações de intubação, encontrados no Hospital Dr. Pedro Garcia Moreno, em Itabaiana, Sergipe. Os grupos foram divididos da seguinte forma:

c.I Grupo Controle (GC): pacientes com COVID-19 não graves (sem a necessidade de ventilação mecânica);

c.II Grupo Teste (GT): pacientes com COVID-19 graves (com a necessidade de ventilação mecânica);

d. Os pacientes do grupo Controle (GC) foram orientados a respeito do estudo e de maneira voluntária, deram o consentimento na pesquisa, através da aplicação do Termo de Consentimento Livre e Esclarecido (TCLE) em anexo ao final do projeto. 
e. Em pacientes que foram entubados, grupo Grupo Teste (GT),Termo de Consentimento Livre e Esclarecido (TCLE), em anexo ao final do presente projeto, foi obtido junto aos seus responsáveis ou familiares.

\subsection{Caracterização do universo e plano de amostragem}

Usando índice de confiança (IC) de 95\% e erro padrão (e) de 5\%, encontra-se um número de 32 pacientes para cada um dos dois grupos e para cada região pesquisada, totalizando um número total ideal de 64 participantes.

\subsubsection{Formação dos grupos}

Grupo Controle (GC): pacientes com COVID-19 não graves (sem a necessidade de ventilação mecânica);

Grupo Teste (GT): pacientes com COVID-19 graves (com a necessidade de ventilação mecânica).

\subsubsection{Critérios de inclusão}

Todos os pacientes selecionados para a pesquisa tiveram diagnóstico laboratorial positivo para COVID-19 (Sars-CoV19), com histórico médico hospitalar, definindo se ficou internado em unidade de terapia intensiva (UTI) ou não, porém permanecendo em ambiente hospitalar, assim como foi definido se é portador ou não de comorbidades como: diabetes, hipertensão arterial, cardiopatias, doenças renais, doenças pulmonares, doenças reumáticas, doenças imunossupressoras, doenças autoimunes, neoplasias malignas com ou sem quimioterapias e pacientes com síndrome de Down.

\subsubsection{Critérios de exclusão}

Paciente com diagnóstico negativo para COVID-19.

\subsubsection{Variável primária}

Presença de comorbidade sistêmica (dados obtidos categoricamente: se sim=1 ou não=0, caso sim, especificar, gerando valores relativos expressos em porcentagem);

Teve febre na COVID-19 (dados obtidos categoricamente: se sim=1, valor de temperatura corporal maior ou igual a $37^{\circ} \mathrm{C}$, ou não $=0$, valor de temperatura corporal menor a $37^{\circ} \mathrm{C}$, gerando valores relativos expressos em porcentagem, mas, caso sim, quantificar em graus Celsius);

Teve hipertensão arterial - HAS (dados obtidos categoricamente: se sim=1, valor da PA maior ou igual a 140 x100 mmhg, ou não=0, valor de PA menor ou igual a $140 \times 100 \mathrm{mmHg}$, gerando valores relativos expressos em porcentagem e média em mmHg);

Falta de ar na COVID-19 (dados obtidos categoricamente: se sim=1 ou não=0, caso sim, especificar também categoricamente se leve, moderada ou forte);

Teve cefaleia na COVID-19 (dados obtidos categoricamente: se $\operatorname{sim}=1$ ou não=0, caso sim, especificar também categoricamente se leve, moderada ou forte);

Teve tosse na COVID-19 (dados obtidos categoricamente: se $\operatorname{sim}=1$ ou não=0, caso sim, especificar também categoricamente em relação à frequência se pouca moderada ou muita);

Teve astenia (moleza) na COVID-19 (dados obtidos categoricamente: se sim=1 ou não=0, caso sim, especificar também categoricamente se leve, moderada ou forte);

Teve disgeusia (perda do paladar) na COVID-19 (dados categóricos: se sim=1 ou não=0, caso sim, responder se já era assim antes); 
Teve anosmia (perda de olfato) na COVID-19 (dados categóricos sim=1 ou não=0, caso sim, responder se já era assim antes);

\subsubsection{Avaliação dos pacientes}

Pacientes com diagnóstico laboratorial de COVID-19 foram avaliados em ambiente hospitalar: os casos graves nas UTIs, com dados consolidados após o conhecimento do tempo de internação em UTI e o tempo de uso da ventilação mecânica; e os casos leves, sem passagem pela UTI, em ambiente ambulatorial, pronto socorro ou enfermaria, porém com os seus dados consolidados apenas com a confirmação posterior da cura, sem ter ocorrido agravamento do quadro clínico. Estes mesmos pacientes responderama um questionário, e prontuários foram consultados para obtenção das seguintes informações, além do nome, data de nascimento/ idade, sexo biológico (masculino ou feminino) e etnia (leucoderma, feoderma ou melanoderma):

Possui comorbidade? Se sim, qual ou quais, se mais de uma, qual a de mais difícil controle?

Teve febre enquanto estava com a Covid-19? Se sim, sabe dizer quantos graus?

Teve falta de ar enquanto estava com a Covid-19? Se sim,forte moderada ou fraca?

Quanto ao paladar, perdeu ou já não era bom?

Quanto ao olfato, perdeu ou já não era bom?

Precisou usar ventilação mecânica (respirador)?

\subsubsection{Riscos}

Existe risco de contaminação pelo SARS-CoV-2, porém todos os cuidados preventivos foram tomados para minimizar o risco de contaminações cruzadas, conforme orientações da SCIH do hospital e da OMS (EPIs utilizados na pesquisa por conta dos pesquisadores).

O risco de revelação das identidades dos pacientes foi minimizado utilizando códigos numéricos nas fichas e formulários utilizados.

\subsubsection{Benefícios}

Investigação das características epidemiológicas da COVID-19.

\subsubsection{Estratégia estatística}

Para resposta da pergunta de pesquisa, foi realizada a média ponderada dos dados qualitativo e quantitativos em cada um dos dois grupos, através destes resultados encontrarem a razão de chances/Oddsration (OR). As relações foram obtidas na razão das médias ponderadas encontradas para os dados quantitativos entre os grupos.

A comparação entre os dois grupos foi realizada pelo teste de Mann Whitney (teste U) para as variáveis numéricas, como a distribuição não foi normal, utilizamos o Shapiro Wilk. Para as variáveis dicotômicas,o teste utilizado foi Mann Whitney.

Os testes foram realizados na versão 6.0 do GraphpadPrim, usando IC=95\% e nível de significância de $5 \%$.

\subsubsection{Aprovação no CEP}

A presente pesquisa obteve aprovação junto ao Comitê de Ética e Pesquisa número CAAE 31540520.2.00005546.

\section{Resultados e Discussão}

Os resultados foram organizados conforme Tabela 1 para melhor discussão e compreensão dos achados. 
Tabela 1 - Resultados demográficos e secundários.

\begin{tabular}{|c|c|c|c|}
\hline Resultados demográficos & Grupo GT & Grupo GC & \\
\hline \multirow[t]{2}{*}{ Idade (média/ SD) } & $59.94(+/-9.93)$ anos de idade & $57.81(+/-11)$ anos de idade & \\
\hline & Maior: 82/ Menor: 39 & Maior: 80/ Menor: 33 & \\
\hline \multirow[t]{2}{*}{ Sexo } & Masculino: $68.25 \%$ (22) & Masculino: $68.75 \%$ (22) & \\
\hline & Feminino: $31.25 \%$ (10) & Feminino: $31.25 \%$ (10) & \\
\hline \multirow[t]{4}{*}{ Etnia } & Feoderma: $43.75 \%$ (14) & Feoderma: $81.25 \%$ (26) & \\
\hline & Leucoderma: $18.75 \%$ (6) & Leucoderma: $3.13 \%$ (1) & \\
\hline & Melanoderma: $3.13 \%$ (1) & Melanoderma: 0\% (nenhum) & \\
\hline & Ignorada: $34.37 \%$ (11) & Ignorada: $15.62 \%(5)$ & \\
\hline Resultados secundários & Grupo GT & Grupo GC & Teste de Mann Whitney \\
\hline \multirow[t]{5}{*}{ Pressão arterial (média/ SD) } & $\begin{array}{c}\text { Sistólica: } 127.19 \mathrm{mmHg} \\
(+/-17.08)\end{array}$ & $\begin{array}{c}\text { Sistólica: } 116.88 \mathrm{mmHg} \\
(+/-12.56)\end{array}$ & $\mathrm{P}=0.0136$ \\
\hline & Diastólica:81.25mmHg & Diastólica: $78.44 \mathrm{mmHg}$ & \multirow{4}{*}{$\mathrm{P}=0.0466$} \\
\hline & $(+/-6.09)$ & $(+/-5.74)$ & \\
\hline & Maior: 160 x $90 \mathrm{mmHg}$ & Maior: 160 x $90 \mathrm{mmHg}$ & \\
\hline & Menor: 100 x $60 \mathrm{mmHg}$ & Menor: 90 x 60 mmHg & \\
\hline Tosse & $84.38 \%(27)$ & $53.13 \%(17)$ & $\mathrm{P}=0.0141$ \\
\hline Dor de Garganta & $3.13 \%(1)$ & $9.38 \%(3)$ & $\mathrm{P}>0.05$ \\
\hline \multirow[t]{3}{*}{ Dificuldade Respiratória } & $84.38 \%(27)$ & $84.38 \%(27)$ & $\mathrm{P}>0.05$ \\
\hline & Muita Dificuldade: & Muita Dificuldade: & \\
\hline & $37,50 \%(12)$ & $9.38 \%(3)$ & $\mathrm{P}=0.0164$ \\
\hline Cefaleia & $78.13 \%(25)$ & $68.75 \%(22)$ & $\mathrm{P}>0.05$ \\
\hline Astenia & $90.63 \%(29)$ & $90.63 \%(29)$ & $\mathrm{P}>0.05$ \\
\hline Disgeusia & $62.50 \%(20)$ & $46.88 \%(15)$ & $\mathrm{P}>0.05$ \\
\hline Diarreia & $6.25 \%(2)$ & $3.13 \%(1)$ & $\mathrm{P}>0.05$ \\
\hline \multirow[t]{2}{*}{ Febre } & Sim: $75 \%$ & Sim: $56.25 \%$ & $\mathrm{P}>0.05$ \\
\hline & Média: $38^{\circ} \mathrm{C}$ & Média: $37^{\circ} \mathrm{C}$ & $\mathrm{P}=0.0266$ \\
\hline \multirow[t]{4}{*}{ Presença de Comorbidades } & Sim: $56.25 \%(18)^{*}$ & Sim: $46.88 \%(15)^{*}$ & $\mathrm{P}>0.05$ \\
\hline & Diabetes: $21.88 \%$ (15) & Diabetes: $9.38 \%$ (13) & $\mathrm{P}>0.05$ \\
\hline & Hipertensão: $44.88 \%$ (7) & Hipertensão: 40.63\% (3) & $\mathrm{P}>0.05$ \\
\hline & *Quatro casos com ambos & * Quatro casos com ambos & \\
\hline
\end{tabular}

DP: Desvio Padrão

Fonte: Autores, com base nos dados coletados

A Tabela 1 mostra os resultados demográficos e secundários encontrados na pesquisa. O número da amostra da pesquisa foi de 64 indivíduos incluídos no processamento estatístico, destes 32 (idade média 59,94 anos) para o grupo GT e 32 (idade média 57,81 anos) no grupo GC; 68,75\% eram do sexo masculino e 31,25\% do sexo feminino para ambos os grupos. Doença grave foi mais prevalente no sexo masculino (Barry et al., 2021). Na presente pesquisa o sexo masculino foi predominante em ambos os grupos (GT e GC). Gómez-Ochoa et al. (2020) encontraram uma maior percentagem de indivíduos com COVID-19 em mulheres (69,98\%) e a média de idade de 40 anos, corroborando com os achados de Martínez et al. (2021) que relataram percentagem de 73,7\% de COVID-19 no gênero feminino e idade média de 45,6 anos. Em contrapartida, Nascimento (2021) e Yang et al. (2020) afirmaram uma maior prevalência em homens em suas pesquisas (53\% e 56\%, 
respectivamente), assim como em nosso estudo. As médias de idade encontradas por Zhang et al. (2020) e Badawi et al. (2021) foram, respectivamente, 39,9 anos e 55 anos de idade, abaixo da média de idade do presente estudo (58,87). Os adultos mostraram mais chance de serem infectados em comparação a crianças (Koh et al., 2020).

Em relação à etnia observa-se que o grupo GC obteve percentagens de feoderma de 81,25\%,3,13\% para leucoderma e 0\% de indivíduos de etnia melanoderma. A percentagem de prontuários com registro ignorado sobre as informações de etnia foi de 15,62\%. No grupo GT, as maiores percentagens em relação às etnias também foram maiores entre pessoas feodermas, apresentando $43,75 \%$ para esta etnia, 18,75\% para a leucoderma e 3,13\% para melanoderma. A percentagem de prontuários com registro ignorado de informações sobre a etnia no grupo GT foi de 34,37\%. A média total de percentagens de anotações ignoradas sobre a etnia foi de $24,99 \%$, chamando a atenção para um maior cuidado com o registro desse importante dado demográfico nos prontuários examinados. Corroborando com nosso estudo, a pesquisa de Kopel et al. (2020) relataram uma maior taxa de infecção e hospitalização dos afro-americanos e hispânicosem relação aos caucasianos, e Mackey et al. (2021) demonstraram maior taxa de infecção pela COVID-19 na população hispânica em relação à população branca.

A percentagem de pessoas do grupo GT que apresentaram febre foi de $75 \%$, e a média de temperatura encontrada no grupo foi de $38^{\circ} \mathrm{C}$. No grupo GC a percentagem de indivíduos com febre foi de $56,25 \%$, mas a média da temperatura corporal no grupo foi de $37^{\circ} \mathrm{C}$, tendo uma média total dos dois grupos de $37,5^{\circ} \mathrm{C}$. Respectivamente, Adil et al. (2020); Kumar et al. (2020); Liguoro et al. (2020); Ludvigsson (2020) e Yang et al. (2020) relataram que o sintoma mais prevalente foi febre (88\%; $89 \%$; 51,6\%; 41,5\% e91.3\%). Deshmukh et al. (2020) e Mehta et al. (2020) também afirmaram que a febre é uma sintomatologia frequente da COVID-19. Barry et al. (2021) relataram média de temperatura corporal de 37,3 $\mathrm{C}$, próxima do valor encontrado em nossa pesquisa.

Os indivíduos dos grupos GT e GC, respectivamente, apresentaram percentagem e frequência significativa de $84,38 \%$ (27) e 53,13\% (17) de sintomatologia de tosse $(\mathrm{P}=0.0141)$. A tosse aparece como a quarta sintomatologia mais frequente em nosso estudo no grupo GT, e a média nos dois grupos foi de 68,75\%. Adil et al. (2020); Kumar et al. (2020) e Yang et al. (2020) encontraram a tosse como a segunda sintomatologia mais frequente, respectivamente com 48,5\%, 68\% e 67.7. Em relação à sintomatologia de dor de garganta os grupos GT e GC apresentaram frequência e percentagem, respectivamente, de $3,13 \%$ (1) e 9,38\% (3), com média de 6,25\%, valores abaixo do encontrado por Kumar et al. (2020); Liguoro et al. (2020) e Ludvigsson (2020), respectivamente, $14 \% ; 17,9$ e 46,2\%.

A dificuldade respiratória encontrada em ambos os grupos foi de 84,38\% (27), registrando grande dificuldade respiratória no GT de 37,50\% (12) e 9,38\% (3) no grupo GC, em contrapartida a dispneia foi menos prevalente nos estudos de Kumar et al. (2020) e Yang et al. (2020),correspondendo, respectivamente, a 19\% e 30,4\%. Em relação às crianças, Liguoro et al. (2020) acrescentaram, ainda, que raramente as crianças necessitaram de respiração auxiliar, sendo que a dispneia estava presente em apenas $7,7 \%$ dos casos.

A frequência e percentagem de indivíduos com cefaleia no grupo GT e GC foi, respectivamente, de 78,13\%(25) e $68,75 \%$ (22). Nosso estudo demonstrou uma prevalência de cefaleia média dos dois grupos (GT e GC) de 73,44\%, valores mais elevados que os estudos de Badawi et al. (2021); Kumar et al. (2020) e Martínez et al. (2021), respectivamente de 10 a 30\%, $14 \%$ e $55,6 \%$.

Ambos os grupos (GT e GC) apresentaram percentagem e frequência de astenia de 90,63\%(29). A astenia foi o sintoma mais prevalente em nossa pesquisa com percentagem de 90,63\%, não concordando com os demais estudos, sendo que, Adil et al. (2020) e Kumar et al. (2020) obtiveram resultados semelhantes de 38\%. O estudo focado em crianças de Ludvigsson (2020) apresentou o resultado mais baixo para astenia de 7,6\%. Os estudos de Martínez et al. (2021); Gómez-Ochoa et al. (2020); Yang et al. (2020) apresentaram resultados para astenia respectivamente de 55,6\%, 43\% e $51 \%$.

A frequência e percentagem de disgeusia encontrada nos grupos GT e GC foram, respectivamente, de 62,50\%(20) e 
46,88(15), sintoma não mensurado pelos autores da bibliografia consultada.

No presente estudo, 6,25\% (2) de indivíduos do grupo GT e 3,13\% (1) do grupo GC apresentaram diarreia. Esta foi presente nos dois grupos (GT e GC) de nosso estudo em 4,69\% dos pacientes, corroborando com o estudo de Kumar et al. (2020) que encontraram valores aproximados de 4\%. A pesquisa de Ludvigsson (2020) demonstrou presença de diarreia em 8,8\% das crianças, e, por fim, os estudos de Barry et al. (2021) e Desmukh et al. (2020) obtiveram resultados, respectivamente, de $37,5 \%$ e $34 \%$ de sintomas gastrointestinais e diarreia.

Outros sintomas como congestão anosmia, nasal, rinorreia, escarro, mialgia, inapetência, dor abdominal e vômito também foram relatados na literatura consultada, porém não encontrados em nossa pesquisa (Deshmukh et al., 2020; Kumar et al., 2020; Yang et al., 2020; Zhang et al., 2020). A ausência de sintomas também é relatada em pacientes hospitalizados por até 11 dias (Walsh et al., 2020).

Quanto às variáveis comorbidades, encontrou-se a percentagem de 56,25\% de indivíduos do grupo GT, sendo 46,88\% com Hipertensão Arterial Sistêmica (HAS) e 21,88\% com Diabetes Melittus (DM). No grupo de pessoas GC, a percentagem foi de $46,88 \%$ com comorbidades, sendo 40,63\% com HAS e 9,38\% com DM. A média dos dois grupos (GT e GC) foi de $43,75 \%$ para HAS e de $15,63 \%$ para DM.

A pressão arterial (PA) média encontrada nos indivíduos do grupo GT foi de 127,19 x 81,25 mmHg e, nos indivíduos do grupo GC, a PA média foi de 116,88 x 78,44, obtendo variação significativa entre os dois grupos, sendo PAS (P=0.0136) e PAD ( $\mathrm{P}=0.0466)$. Resultados semelhantes de PA sistólica foram encontrados por Barry et al. (2021) de 118mmHg.

Há aumento da mortalidade na COVID-19 em pacientes com comorbidades, entre elas DM, HAS e outras doenças cardiovasculares. Os pacientes com hipertensão tiveram a maior taxa de mortalidade com 44\%, seguido de DM (24,1\%) e doença coronária (14,8\%) (Deshmukh et al., 2020).

As comorbidades mais prevalentes nos trabalhos de Barry et al. (2021); Gómez-Ochoa et al. (2020); Yang et al. (2020) foram HAS ( $43 \%, 7 \%$ e $21 \%$ ) e DM ( $43 \%, 4 \%$ e 9,7\%,), corroborando com nossos achados, em contrapartida, o estudo de Nascimento (2021) obteve a doença coronariana como a comorbidade mais frequente de $65 \%$. Os casos severos no estudo consistiram em pacientes mais velhos com comorbidades, demonstrando que a idade e as comorbidades são fatores de risco (Yang et al., 2020).

Segundo Liguoro et al. (2020), raramente crianças necessitaram de suporte respiratório, sendo que dispneia estava presente em apenas 7,7\% dos casos. Em nossa pesquisa não encontramos crianças no período de avaliação.

Kumar et al. (2020) afirmaram que os pacientes com comorbidades como DM, HAS, obesidade, doença cardiovascular, câncer, doença respiratória crônica e doença hepática possuem maior risco de mortalidade, enquanto que Adil et al. (2020); Badawi et al. (2021); Madwellet et al. (2020); Salzberger et al. (2021) adicionaram que idades avançadas e imunossupressão também são fatores de risco.

Em nossa pesquisa não houve registro de imunossupressão, obesidade, doença cardiovascular, câncer, doença respiratória crônica e hepática.

\section{Conclusão}

Diante da literatura consultada e dos resultados encontrados, conclui-se que as idades médias do GT e GC foram aproximadas, respectivamente, de 59,94 anos e 57,81 anos, apresentando uma idade média geral dos dois grupos de 58,9 anos. Além disso, o sexo masculino foi o mais frequente entre os indivíduos com COVID-19 nos dois grupos. A etnia mais encontrada nos grupos GT e GC foi a feoderma, seguido da leucoderma. As percentagens de prontuários com registro ignorado sobre a etnia foram elevadas nos grupos pesquisados.

Em relação à sintomatologia, a percentagem de pessoas do grupo GT que apresentaram febre foi mais elevada, assim 
como a média de temperatura corporal foi mais elevada, os indivíduos dos grupos GT obtiveram maior frequência de tosse, apresentando-se como a quarta sintomatologia mais frequente em nosso estudo. A astenia foi a sintomatologia mais frequente em nosso estudo. O grupo GC relatou mais percentagem de dor de garganta. A frequência de indivíduos com cefaleia, diarreia e disgeusia foi maior no grupo GT.

A PA média foi mais elevada no grupo GT, enquanto a percentagem de dificuldade respiratória encontrada em ambos os grupos foi idêntica, porém, na avaliação da gravidade, o grupo GT apresentou maior registro de grande dificuldade respiratória. As comorbidades mais frequentemente encontradas nos dois grupos foram HAS e DM, entretanto houve uma maior incidência dessas doenças no grupo GT.

Sugere-se realização de mais pesquisas para elucidações das características clínicas e possíveis envolvimentos de comorbidades na COVID-19.

\section{Referências}

Adil, M. T., Rahman, R., Whitelaw, D., Jain, V., Al-Taan, O., Rashid, F., Munasinghe, A., \& Jambulingam, P. (2021). SARS-CoV-2 and the pandemic of COVID-19. Postgrad Med J, 97(1144), 110-116. https://doi.org/10.1136/postgradmedj-2020-138386

Badawi, A., \& Vasileva, D. (2021). Comparative profile for COVID-19 cases from China and North America: Clinical symptoms, comorbidities and disease biomarkers. World J ClinClin Cases, 9(1), 118-132. https://doi.org/10.12998/wjcc.v9.i1.118

Barry, M., Althabit, N., Akkielah, L., AlMohaya, A., Alotaibi, M., Alhasania, S., Aldrees, A., AlRajhi, A., AlHiji, A., Almajid, F., AlSharidi, A., Al-Shahrani, F. S., Alotaibi, N. H., \& AlHetheel, A. (2021). Clinical characteristics and outcomes of hospitalized COVID-19 patients in a MERS-CoV referral hospital during the peak of the pandemic. International journal of infectious Diseases, (106), 43-51. https://doi.org/10.1016/j.ijid.2021.03.058

Bulfone, T. C., Malekinejad, M., Rutherford, G. W., \& Razani, N. (2020). Outdoor Transmission of SARS-CoV-2 and Other Respiratory Viruses: A Systematic Review. The Journal of Infectious Diseases, 223(4), 550-561. https://doi.org/10.1093/infdis/jiaa742

Deshmukh, V., Tripathi, S., Pandey, A., Deshmukh, V., Vykoukal, J., Patil, A., \& Sontakke, B. (2020). COVID-19: a conundrum to decipher. European Review for Medical and Pharmacological Sciences, 24(10), 5830-5841. https://doi.org/10.26355/eurrev_202005_21378

Gómez-Ochoa, S. A., Franco, O. H., Rojas, L. Z., Raguindin, P. F., Roa-Díaz, Z. M., Wyssmann, B. M., Guevara, S. L. R., Echeverría, L. E., Glisic, M., \& Muka, T. (2020). COVID-19 in Health-Care Workers: A Living Systematic Review and Meta-Analysis of Prevalence, Risk Factors, Clinical Characteristics, and Outcomes. Am J Epidemiol, 190(1), 16-175. https://doi.org/10.1093/aje/kwaa191

Koh, W. C., Naing, L., Chaw, L., Rosledzana, M. A., Alikhan, M. F., Jamaludin, S. A., Amin, F., Omar, A., Shazli, A., Griffith, M., Pastore, R., \& Wong, J. (2020). What do we know about SARS-CoV-2 transmission? A systematic review and metaanalysis of the secondary attack rate and associated risk factors. PLOS ONE, 15(10), e0240205. https://doi.org/10.1371/journal.pone.0240205

Kopel, J., Perisetti, A., Roghani, A., Aziz, M., Gajendran, M., \& Goyal, H. (2020). Racial and Gender-Based Differences in COVID-19. Frontiers in Public Heath, (8). https://doi.org/10.3389/fpubh.2020.00418

Kumar, M., Taki, K., Gahlot, R., Sharma, A., \& Dhangar, K. (2020). A chronicle of SARS-CoV-2: Part-I - Epidemiology, diagnosis, prognosis, transmission and treatment. Science of the Total Environment, (734), 139278. https://doi.org/10.1016/j.scitotenv.2020.139278

Khan, M., Adil, S. F., Alkhathlan, H. Z., Tahir, M. N., Saif, S., Khan, M., \& Khan, S. T. (2020). COVID-19: A Global Challenge with Old History, Epidemiology and Progress So Far. Molecules, 26(1), E39. https://doi.org/10.3390/molecules26010039

Li, L., Li, R., Wu, Z., Yang, X., Zhao, M., Liu, J., \& Chen, D. (2020). Therapeutic strategies for critically ill patients with COVID-19. Annals of Intensive Care, 10(1), 45. https://doi.org/10.1186/s13613-020-00661-z

Liguoro, I., Pilotto, C., Bonanni, M., Ferrari, M. E., Pusiol, A., Nocerino, A., Vidal, E., \& Cogo, P. (2020). SARS-COV-2 infection in children and newborns: a systematic review. European Journal of Pediatrics, 179(7), 1029-1046. https://doi.org/10.1007/s00431-020-03684- 7

Ludvigsson, J. F. (2020). Systematic review of COVID-19 in children shows milder cases and a better prognosis than adults. Acta Paediatrica, 109(6), 10881095. https://doi.org/10.1111/apa.15270

Madewell, Z. J., Yang, Y., Longini Jr, I. M., Halloran, M. E., \& Dean, N. E. (2020). JAMA Network Open, $3(12)$, e2031756. https://doi.org/10.1001/jamanetworkopen.2020.31756

Mackey, K., Ayers, C. K., Kondo, K. K., Saha, S., Advani, S. M., Young, S., Spencer, H., Rusek, M., Anderson, J., Veazie, S., Smith, M., \& Kansagara, D. (2020). Racial and Ethnic Disparities in COVID-19-Related Infections, Hospitalizations, and Deaths. Annals of Internal Medicine, 174(3), 362-373. https://doi.org/10.7326/M20-6306

Martínez, I. P., Torres, L. A. P. d., Lama, J. G., García, C. J., Montero, R. S., \& Garrido, F. R. (2020). Características clínico-epidemiológicas de la infección por el virus SARS-CoV-2 en médicos de familia: un estudio de casos y controles. Atencion Primaria, (53), 101956. https://doi.org/10.1016/j.aprim.2020.12.001 
Mehta, N. S., Mytton, O. T., Mullins, E. W. S., Fowler, T. A., Falconer, C. L., Murphy, O. B., Langenberg, C., Jayatunga, W. J. P., Eddy, D. H., \& NguyenVan-Tam, J. S. (2020). SARS-CoV-2 (COVID-19): What Do We Know About Children? A Systematic Review. Clinical Infectious Diseases, 71(9), 24692479. https://doi.org/10.1093/cid/ciaa556

Nascimento, M. d. M. (2021). Clinical characteristics of 1544 Brazilians aged 60 years and over with laboratory evidence for SARS-CoV-2. Archives of Gerontology and Geriatrics, (96), 104462. https://doi.org/10.1016/j.archger.2021.104462

Raghuvamsi, P. V., Tulsian, N. K., Samsudin, F., Qian, X., Purushotorman, K., Yue, G., Kozma, M. M., Hwa, W. Y., \& Lescar, J. (2021). SARS-CoV-2 S protein:ACE2 interaction reveals novel allosteric targets. Biochemistry and Chemical BiologyStructural Biology and Molecular Biophysics, (10), e63646. https://doi.org/10.7554/eLife.63646

Rodrigues, M. d. B., Chong-Silva, D. C., \& Neto, H. J. C. (2021). Guia prático para produção e submissão de artigos. Sociedade Brasileira de Pediatria, (5). Salzberger, B., Buder, F., Lampl, B., Ehrenstein, B., Hitzenbichler, F., Holzmann, T., Schmidt, B., \& Hanses, F. (2020). Epidemiology of SARS-CoV-2. Infection, 49(2), 233-239. https://doi.org/10.1007/s15010-020-01531-3

Walsh, K. A., Jordan, K., Clyne, B., Rohde, D., Drummond, L., Byrne, P., Ahern, S., Carty, P. G., O’Brien, K. K., O’Murchu, E., O’Neill, M., Smith, S. M., Ryan, M., \& Harrington, P. (2020). SARS-CoV-2 detection, viral load and infectivity over the course of an infection. Journal of infection, 81(3), 357-371. https://doi.org/10.1016/j.jinf.2020.06.067

Yang, J., Zheng, Y., Gou, X., Pu, K., Chen, Z., Guo, Q., ji, R., Wang, H., Wang, Y., \& Zhou, Y. (2020). Prevalence of comorbidities and its effects in patients infected with SARS-CoV-2: a systematic review and meta-analysis. International Journal of infectious Diseases, (94), 91-95. https://doi.org/10.1016/j.ijid.2020.03.017

Zhang, X., Huang, H., Zhuang, D., Nasser, M. I., Yang, M., Zhu, P., \& Zhao, M. (2020). Biological, clinical and epidemiological features of COVID-19, SARS and MERS and AutoDock simulation of ACE2. Infectious Diseases of Poverty, 9(1), 99. https://doi.org/10.1186/s40249-020-00691-6 